\title{
BMJ Open Qualitative study to conceptualise a model of interprofessional collaboration between pharmacists and general practitioners to support patients' adherence to medication
}

\author{
Adam P Rathbone, ${ }^{1,2}$ Sarab M Mansoor, ${ }^{1}$ Ines Krass, ${ }^{1}$ Kim Hamrosi, ${ }^{1}$ \\ Parisa Aslani ${ }^{1}$
}

To cite: Rathbone AP, Mansoor SM, Krass I, et al. Qualitative study to conceptualise a model of interprofessional collaboration between pharmacists and general practitioners to support patients' adherence to medication. BMJ Open 2016;6:e010488. doi:10.1136/bmjopen-2015010488

- Prepublication history and additional material is available. To view please visit the journal (http://dx.doi.org/ 10.1136/bmjopen-2015010488).

APR and SMM are joint first authors.

Received 14 November 2015 Revised 9 February 2016 Accepted 22 February 2016

CrossMark

\footnotetext{
${ }^{1}$ Faculty of Pharmacy, The University of Sydney, Sydney, New South Wales, Australia ${ }^{2}$ School of Medicine, Pharmacy and Health, University of Durham, Stockton-on-Tees, UK
}

Correspondence to Professor Parisa Aslani; parisa.aslani@sydney.edu.au

\section{ABSTRACT}

Objectives: Pharmacists and general practitioners (GPs) face an increasing expectation to collaborate interprofessionally on a number of healthcare issues, including medication non-adherence. This study aimed to propose a model of interprofessional collaboration within the context of identifying and improving medication non-adherence in primary care.

Setting: Primary care; Sydney, Australia.

Participants: 3 focus groups were conducted with pharmacists $(n=23)$ and 3 with GPs $(n=22)$ working in primary care.

Primary and secondary outcome measures: Qualitative investigation of GP and pharmacist interactions with each other, and specifically around supporting their patients' medication adherence. Audiorecordings were transcribed verbatim and transcripts thematically analysed using a combination of manual and computer coding.

Results: 3 themes pertaining to interprofessional collaboration were identified (1) frequency, (2) co-collaborators and (3) nature of communication which included 2 subthemes (method of communication and type of communication). While the frequency of interactions was low, the majority were conducted by telephone. Interactions, especially those conducted face-to-face, were positive. Only a few related to patient non-adherence. The findings are positioned within contemporary collaborative theory and provide an accessible introduction to models of interprofessional collaboration.

Conclusions: This work highlighted that successful collaboration to improve medication adherence was underpinned by shared paradigmatic perspectives and trust, constructed through regular, face-to-face interactions between pharmacists and GPs.

\section{INTRODUCTION}

Medication non-adherence remains a significant issue for patients, prescribers and

\section{Strengths and limitations of this study}

- This study is the first to have qualitatively investigated mediators of interprofessional collaboration between community pharmacists and general practitioners (GPs) to support patients' adherence to medications in primary care.

- Secondary thematic analysis was carried out on transcribed data without a priori knowledge of the focus group discussions, and analysis was conducted using a combination of manual and computer coding to ensure robust data analysis.

- Participants were recruited via a market research company from a pool of GPs and community pharmacists who had agreed to take part in research. The findings are limited in generalisability to the broader pharmacist and GP populations.

healthcare systems across the globe $^{1}$ Interprofessional collaboration is a growing trend that sees health practitioners from different backgrounds coming together to offer patient services or interventions. ${ }^{23}$ Complex interventions to improve adherence are often based within a collaborative interprofessional setting, yet little is known about how the collaboration functions and the opinions of those involved. ${ }^{1}{ }^{4}$ Currently therefore there is no formal structure in primary care to facilitate patient-prescriber collaboration in identifying and addressing nonadherence in patients on chronic therapy.

The only structured form of pharmacist collaboration in Australia is seen in the Home Medicines Review (HMR). An HMR may be initiated for an at-risk patient by referral from a general practitioner (GP) to an accredited HMR pharmacist who then visits the patient in their home to conduct an 
interview during which the patients' medicines are discussed and inspected. After the review, the pharmacist prepares a report for the patient's GP with recommendations for improving medicine management. The pharmacist and GP should discuss the findings in the report, and the GP should develop a medicine management plan for the patient. In practice, research has shown that the final step of communication between pharmacist and GP is often omitted. Moreover, the accredited pharmacist conducting the review may be independently contracted by the GP. ${ }^{5}$

Several conceptual models of interprofessional collaboration between physicians and pharmacists have been developed. ${ }^{6-16}$ An American model of collaborative working relationship was proposed by McDonough and Doucette. ${ }^{6}$ Their model of collaboration describes 'context characteristics' that is, the environmental setting of professional interactions, 'individual characteristics' relating to the personality and demographics of collaborators, and 'exchange characteristics' which includes trustworthiness, roles, relationship initiations and 'norm development'. ${ }^{6}$ McDonough and Doucette's model was expanded by Bradley $e t a l^{7}$ based on qualitative interviews with GPs and community pharmacists. Their approach describes a multilayered model of integration, and stratifies collaborations according to seven mediators ('locality', 'service provision', 'trust', 'knowing each other', 'communication', 'professional roles' and 'professional respect'). In Hudson's model (cited in Leathard (1994) $)^{9}$ four pillars of interprofessional collaboration are conceptualised and include 'communication', 'coordination', 'co-location' and 'commissioning'. Hudson argued that multiple levels of collaboration existed between collaborators, with collaborators moving through stages of collaboration. Hudson's work dichotomises collaboration in optimistic and pessimistic models, concluding that 'the scope of professional integration is greater than tends to be assumed' suggesting there are many opportunities for collaboration. ${ }^{10} \mathrm{~A}$ model developed by Van $e t a l^{1-14}$ describes collaboration from the perspective of the pharmacist and physician, citing 'interactional', 'environmental' and 'practitioner determinants' as predictors of collaboration and highlighting the importance of frequent or historical interactions during training years.

Hughes and McCann ${ }^{15}$ identified barriers to interprofessional collaboration in Northern Ireland. They identified a key barrier as the 'shopkeeper' image of pharmacists, arguing that the conflict between providing health services and running a business limited the willingness of GPs to collaborate with pharmacists. The gatekeeping role of GP secretaries was also identified as barrier to collaboration. ${ }^{15} \mathrm{~A}$ recent review by Bardet et $a l^{17}$ concluded additional core determinants of 'trust', 'perceptions and expectations of other healthcare professionals' were essential for collaboration, highlighting the importance of the physicians' social perception of the pharmacist. Howard et $a l^{16}$ identified 'role expectation' as an issue for family physicians and pharmacists who took part in a trial in which they worked together to optimise medicines management for patients over 65 years. The professionals involved in the trial had differing perspectives of what pharmacists were able to do-suggesting shared perspectives may be the key to developing trust and successful collaboration.

As far as the authors are aware, models of interprofessional collaboration have not been presented within the context of improving medication adherence. A recent review concluded that behavioural interventions delivered by multiprofessional teams 'offered the best opportunity to improve adherence'. ${ }^{4}$ As such, this study aimed to conceptualise a model of interprofessional collaboration within the context of improving medication adherence.

\section{METHODS}

A qualitative study using focus group discussions with GPs and community pharmacists was undertaken to address the study aim. The participants were registered pharmacists in community practice and GPs, over the age of 21 years and who spoke English fluently.

\section{Recruitment}

To maximise the number of respondents, community pharmacists and GPs were recruited using a market research company (J\&S Research Services, Cremorne, Australia). The company has a database of pharmacists and GPs who have agreed to take part in research. However, a response rate (ie, how many were contacted and how many refused to participate) was not recorded by the company. Participants were reimbursed for their involvement in the focus groups at $\$ 150$ for the pharmacists and $\$ 200$ for GPs, based on current rates used by the market research company. Forty-eight eligible participants were recruited, with 45 participating in six focus groups, between June 2013 and February 2014. Participant demographics are shown in table 1.

\section{Focus groups}

Three focus groups were conducted with GPs and three focus groups were conducted with pharmacists working in primary care in Sydney, Australia. Each focus group discussion consisted of 6-8 participants, and lasted for approximately $1.5 \mathrm{~h}$. Owing to the qualitative nature of this study, focus group discussions were conducted until 'theoretical data saturation' had been reached. ${ }^{18}$

Focus group discussions were conducted using a semistructured protocol to address the study aims (see online supplementary appendix 1 ). The broad topics covered in the discussions included GPs' and community pharmacists' awareness and understanding of their patients' adherence and non-adherence to medications, current adherence interventions/services delivered by 
Table 1 Participant demographics

\begin{tabular}{lll}
\hline $\mathbf{n = 4 5}$ & GPs & Pharmacists \\
\hline Total & 22 & 23 \\
$\begin{array}{l}\text { Female } \\
\text { Male }\end{array}$ & 12 & 13 \\
$\begin{array}{l}\text { Spoke English as first } \\
\text { language (at home) }\end{array}$ & 10 & 10 \\
$\begin{array}{l}\text { Language other than English } \\
\text { as first language }\end{array}$ & 6 & 14 \\
$\begin{array}{l}\text { Born in Australia } \\
\text { Born overseas }\end{array}$ & 8 & 9 \\
$\begin{array}{l}\text { Education level } \\
\text { Merking hours/week }\end{array}$ & 14 & 12 \\
& Tertiary 3 & Tertiary 11 \\
& BPharm 14 & MBBS 9 \\
& MPharm 5 & Bachelor 2 \\
Mean ( \pm SD) & Missing 1 & \\
Median (IQR) & $36.6( \pm 9.6)$ & $43.0( \pm 10.8)$ \\
\hline
\end{tabular}

participants in their practice; and their experience of and opinions about an interprofessional approach to support adherence. The face and content validity of the interview protocol was established through pretesting of the protocol with a small sample of researchers who were also pharmacists $(n=3)$. Focus groups were conducted by the researchers (KH (lead facilitator), PA, IK and SMM), and handwritten notes were taken during the focus groups (SMM). For the purposes of this study, collaboration was defined as working together to identify non-adherence and factors contributing to nonadherence in patients on chronic therapy, and supporting their adherence to their medications.

\section{Data analysis}

Focus groups were audio-recorded with permission of the participants, transcribed verbatim and transcripts quality checked for accuracy. The validity of the findings is embedded within the methodological approach to the analysis. Secondary thematic analysis of the rich, qualitative data was carried out without a priori knowledge of the focus groups or primary analysis to ensure validity, rigour and avoid bias. The secondary thematic analysis was conducted by an independent researcher (APR) who did not participate in the focus groups using a combination of manual coding and NVivo V.10 computer software (QSR International: Melbourne, Australia). Analysis was conducted using a typical method of constant comparison to identify new codes and merge similar ones. ${ }^{18}$ Similar codes were then clustered together to form themes. Each theme was analysed within the context of current interprofessional collaboration models outlined above, to identify similarities and differences in terminology and concepts to highlight which model and/or mediators functioned as facilitators or barriers to collaboration to improve adherence. Each participant attending each session was assigned a code. For example, participants in each focus group were coded as F1=female 1, F2=female 2, M3=male 3 and so on.

\section{RESULTS}

A total of 45 respondents participated in the focus groups (table 1), 22 GPs in three focus groups and a further 23 pharmacists in another three focus groups.

Three main themes were identified as mediators of interprofessional collaboration within the context of improving adherence. Theme 1-frequency of contact, describes the regularity with which professionals interacted, which appeared to be influenced by patients accessing 'multiple pharmacists and multiple doctors', rather than a regular pharmacist and a regular doctor. Theme 2-co-collaborators, relates to perceptions of the credibility of collaborative partners, co-location and gatekeeper issues. The final theme 'nature of communication' amalgamates two subthemes, (1) the method of communication including written, electronic, telephone or face-to-face, and (2) the type of communication, reactive or proactive. Figure 1 shows the identified themes (left) with the associated functions of successful collaboration (in the middle) through engendering shared perspectives.

Theme 1 -frequency of contact

Respondents described the frequency of interactions with each other as critical to successful collaboration to improve adherence. They believed there were insufficient collaborative interactions with their GP or pharmacist counterpart that were focused on clinical issues, such as medication adherence. Participants also reported that patients' frequent access to a regular pharmacist and a regular doctor was essential for healthcare professional collaboration to identify and intervene where adherence issues were evident.

...I try to encourage them to at least have one pharmacist that knows their history, especially when they've got chronic medical conditions that can advise them on this and that and the other. And I know that pharmacist is going to pick up the phone and call me. "[Name of GP], do you realise Mrs Smith is taking this...". Focus group (FG)3 General Practitioner (GP) Male (M) 1

Like how do you monitor every single person who does go into the door, and they don't even know if they're the regular customers or notice if they're regular customers, yeah. You can't...it is impossible to track, I think, adherence even if you have meetings. Like what are you going to talk about? My patients may not see you. Your patients may not come to us. So really that only works if your customers come and see us regularly. FG6 Pharmacist $(\mathrm{Ph}) \mathrm{F} 2$

Some respondents indicated they had good relations with their local doctors or pharmacists that were mediated by regular contact, and were willing to 


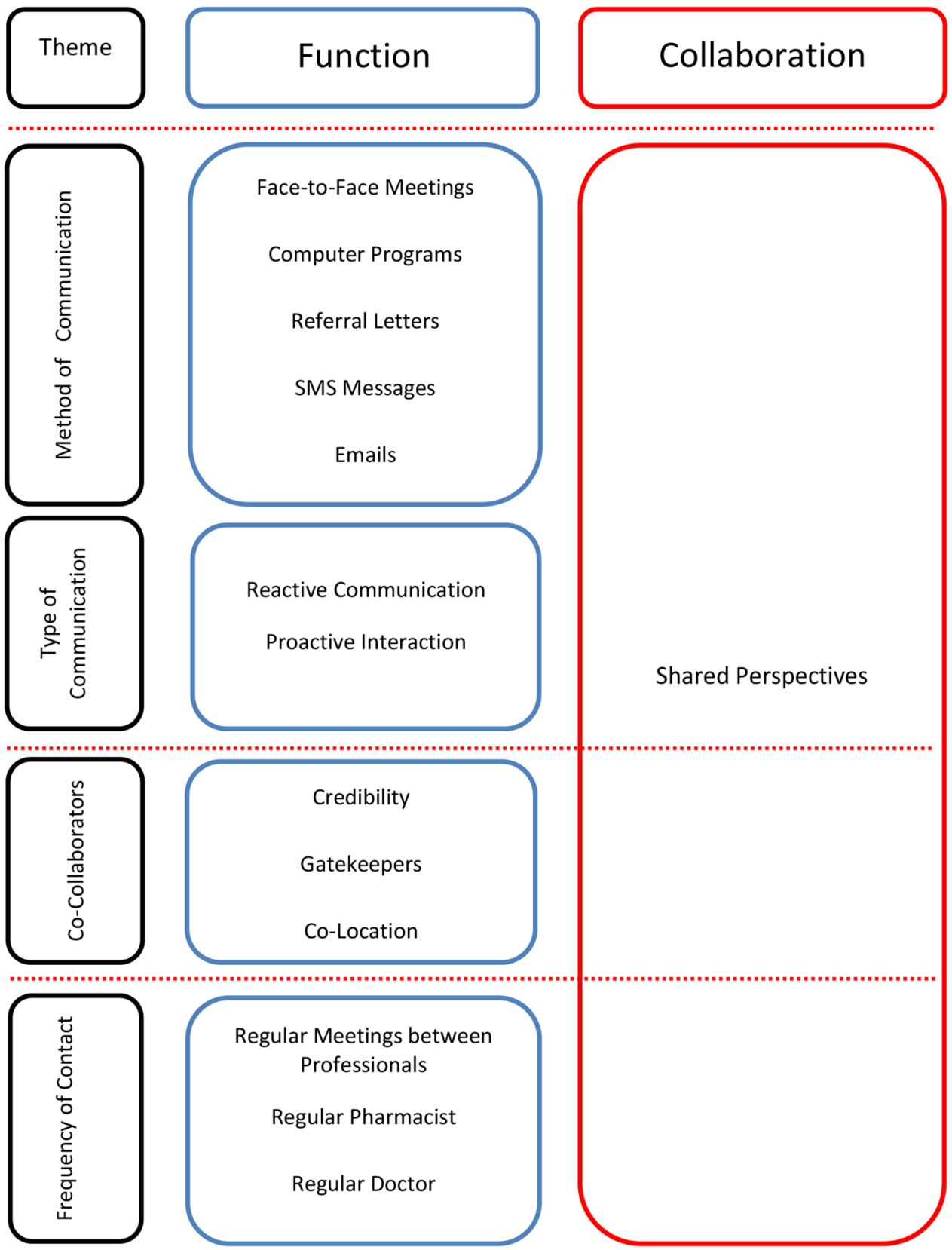

Figure 1 Model of interprofessional collaboration to identify and improve non-adherence.

collaborate with each other to improve patients' adherence to medications.

Well look, I've got a good relationship with the pharmacists that work around my area, notwithstanding that sometimes they have a pharmacy... a different pharmacist come $[\mathrm{s}]$ in and help[s] them out, but the ones that are there regularly I know quite well and I'm quite happy to pick up the phone and talk to them about patients' prescriptions and sometimes I will ring them and check to see if they've actually been dispensed as the patient says or tells me, just to double check. FG5 GP M1

...we have quite a close working relationship with the doctors, say if I've done a MedsCheck [a face-to-face consultation with a consumer, conducted by a pharmacist, aimed at improving medication use] and part of it he will do a referral letter I will also follow it up with a phone call...After that I will tell the customer to come in and then they might make the change once the customer is there with them... FG2 Ph F6

The need for frequent interactions between patients and healthcare professionals also applied to collaboration with other medical professionals, such as specialists, and primary healthcare teams, as well as with the patient's carer.

There is a mental health care team especially like the Webster [dose administration aid] patients who are young, who are on medication, [one] needs to look at 
adherence quite closely. So the mental team is someone who we would get in contact regularly if there's any issues so they will communicate which helps a lot dealing with the customers. FG1 Ph F4

Overall, interactions between pharmacists and GPs were infrequent and irregular, and predominantly focused on issues not related to adherence.

I get calls from them fairly frequently when...I guess if there's a concern with the compliance with the medication or if they come in and there's a long period between say the finishing of one script packet and the next one being dispensed-they'll call me, but most of the calls are...to be honest, most of the calls we get or I get from pharmacists are pharmacists that don't know the patient. They're just a new patient to them. 'I just wanted to make sure if this script is correct?' That goes back to my point about having a regular pharmacist. FG5 GP M1

However, when interactions were more frequent, GPs and pharmacists shared similar perspectives and trusted one another, demonstrated by becoming more comfortable communicating out of hours to ask for favours based on mutual understanding. This enabled them to address clinical issues collaboratively.

There's [sic] a few other doctors that we do chat on a regular basis to the point where they don't want us to call them doctors or by their surname. We have got each other's mobile phones [numbers]. It's to the point where they can call me on a Sunday night and can ask me for something or do a favour for them. That's the level it's gone to. FG6 Ph M2

\section{Theme 2-co-collaborators}

Credibility, co-location and gatekeepers were issues that mediated with whom and when effective collaboration could occur to improve adherence. For example, GPs questioned the credibility of alternative healthcare practitioners with different paradigmatic perspectives to healthcare, such as naturopaths, whose healthcare perspective and approach differed from traditional western medicine and did not consider them suitable collaborators in patient care. It appeared that where pharmacists and GPs did not share similar perspectives, about each other's roles and goals in relation to adherence, collaboration was more difficult as it lacked trust. As pharmacists and GPs were exposed to each other, through co-location, perspectives were renegotiated and aligned leading to shared perspectives and trust.

I would have to agree it would be with people that I felt their qualifications were really legitimate, hard earned and stood up. I would not want a collaboration with all the others it's just going to turn into a dog fight but it's going to turn into not a productive conversation in my mind, I might be totally wrong but they'll be coming from there and we'll be coming from here and I don't see much common ground ever being established because they just want to promote their way-well that's as I see it anyway. FG4 GP F3

Overall, pharmacists were regarded as credible healthcare professionals by most of the GP participants. Pharmacists took a broader view and reported multiple co-collaborators as credible to identify and improve nonadherence, including the patient and carers. Pharmacists felt that given their more frequent contact (theme 1) with the patient/carer that they could perform, and were responsible for adopting, an independent or gatekeeper role when collaborating to identify or improve adherence. They reported that they saw patients on chronic therapy more frequently than other healthcare professionals, including GPs. The impact this has on the nature of communication between co-collaborators is expanded on in theme 3 .

If they don't comply, they don't adhere, they're not even seeing the doctor... If you talk to the patient directly it's more useful. Even talking to the carer, talking to the sister, talking to the mother, talking to the son are much more helpful because you have immediate impact. FG1 $\mathrm{Ph} \mathrm{M1}$

I totally agree with that like we need to have a good interprofessional relationship even though we still need to have a good relationship with our patients and our carers and families... FG1 Ph F4

Collaboration was mediated by co-location and gate keepers. From the GP participants' perspective, their gate keeping role had been significantly eroded with patients collaborating independently with multiple practitioners, therefore limiting the GP's ability to collaborate with 'a pharmacist' to identify and intervene with non-adherence.

On the other hand, pharmacists felt that secretaries acted as gatekeepers and reported experiences of ineffective collaboration.

A lot of the problem with the specialists is the receptionist. You can't get past them. Seriously, they will not let you speak to them. FG6 Ph F1

However, co-location was reported as a significant facilitator of collaboration and overcoming access issues to GPs. When gatekeeping secretaries inhibited collaboration, the close proximity of the GP surgery allowed gatekeepers to be bypassed.

Well there is one doctor just across the road from us. I can never get access to him because like you said the receptionist just blocks me, so one day I just went to the doctor's surgery and I actually knocked on the door, obviously after the patient came out. And the doctor actually let me in and we had a sat down for about ten minutes discussing the patient, so I bypassed that receptionist but that's only because he's across the road. FG6 Ph M1 
Theme 3-nature of communication

A theme relating to both frequency of contact and co-collaborators was the nature of communication. This was characterised by two subthemes. Subtheme (1) method of communication, relates to how co-collaborators actually communicated. Multiple means of communication were utilised for interprofessional collaboration. These included written, electronic, telephonic and face-to-face. Participants reported written, electronic communication as effective for minor or routine interaction or information exchange, but all participants supported face-to-face communication as best for cultivating effective collaboration.

...if there is ever a problem they do actually come out of their office, go to the pharmacy, face to face contact and they say, "Can you make this up? Can you give me suggestions?" And that definitely helps a lot... FG3 Ph F2.

Subtheme (2) type of communication emerged as both professional groups reported a high incidence of reactive communication, that is, contacting one another when faced with a problem, and only few examples of proactive communication, that is, routine meetings. Reactive communication engendered a theme of 'responsibility' where pharmacists would contact GPs reluctantly as from the pharmacists' perspective adherence issues were something pharmacists were responsible for dealing with, leading to a reluctant nature of communication with GPs.

It's not very common that we contact the doctor about their adherence at all. I mean imagine calling the doctor and saying this person is not really compliant with their medication. Then the next step the doctor is going to go 'what do you want me to do about it'. I think it's more so our job description rather than the doctors job description to make sure they're compliant. I mean obviously we're more accessible to them and when doctors give them five repeats they're not going to go back to the doctor in six months' time, so we see them more than they see the doctor. FG6 Ph F2

The quote above illustrates the barrier to collaboration that exists when pharmacists and GPs do not share the same perspectives about roles and responsibilities for improving patients' adherence.

Interestingly, remuneration was raised as a potential strategy. GP participants suggested that the availability of remuneration would allow a dedicated interprofessional adherence support strategy to be developed and implemented in the community setting.

...I suppose one of the things would be if you got paid for doing that then you could probably dedicate a bit more time to it...So to be able to do the things that you are talking about here if there was some form of reimbursement that could happen... FG2 GP M3
Participants suggested, rather than reactive communication, proactive interactions facilitated by local organisations enabled collaboration due to their informal or social, face-to-face nature.

I'm with what we call a Vietnamese Health Professional Association which with doctors, dentists, allied health pharmacists. And it's an organisation that started up since ' 89 and we have functions and things like that, so it involves relationships with all these allied health professionals. I think to be in the area with all of them it helps because you've got that good relationship between healthcare professionals and an understanding. Now working outside of their area it could be a bit difficult because you don't get in contact with them much, but I find that very helpful and we do have a strong association. FG1 Ph M2

If we had face to face and occasionally that does happen. I've been to psychiatric meetings and there are chemists, the local chemist has turned up, same with physios [sic] and sports orthopaedic meetings. So that actually is a good thing because you meet them face to face and I think that personal touch does help you know to kind of engender respect and credibility in the other person. FG4 GP F1

The quote above demonstrates how exposure to one another changes perspectives of credibility and respect, building trust. When perspectives are aligned and shared, GPs and pharmacists come to trust one another's ability to improve adherence, and collaboration was described as being easier.

Using the themes above a model was constructed (figure 1) to present how the identified codes interacted and mediated interprofessional collaboration to improve adherence (figures 2 and 3). The findings point towards a process of socialisation between pharmacists and GPs, where relationships and shared perspectives are socially constructed, resulting in trust and facilitating collaboration (see Discussion)

\section{DISCUSSION}

Overall, this study has indicated that from the perspectives of pharmacists and GPs, successful collaboration was characterised by regular, face-to-face proactive interactions (figure 2) while poor collaboration occurred when interactions were irregular and infrequent (figure 3). The findings therefore support an interprofessional model of collaboration that is mediated by regular, face-to-face interactions. This appeared to be underpinned through trust, embodied as shared perspectives of healthcare, adherence, professional roles and goals, credibility and respect; influencing the nature and frequency of communication. Participants reported written, computer-aided communication might also be helpful for collaboration where regular, face-to-face interaction is not possible. Co-location enabled regular, face-to-face relationship building, which facilitated conversation and 
Figure 2 Facilitators of successful collaboration to identify and improve non-adherence.

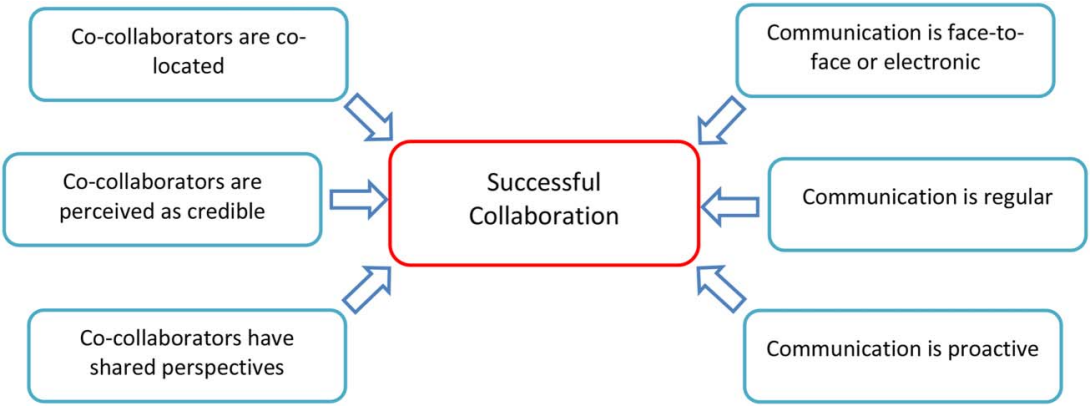

exposure, allowing preconceived perspectives to be renegotiated and trust developed. This enhanced communication and information exchange between the professional groups, improving the identification of nonadherence and enabling collaborative improvement strategies to be implemented.

This work supports the model put forward by Bradley et $a l^{7}$ with its emphasis of the importance of 'regularity' in relation to pharmacist and GP interactions with each other and patients. Without regular interaction, pharmacists and GPs reported difficulty in identifying nonadherence through an unwillingness to share information with co-collaborators or communicate. There are several similarities between the mediators identified in their model and those identified in this study, for example, 'locality' or co-location of collaborators. ${ }^{78}$ This study identified co-location as a strategy to bypass gatekeepers and increase direct, face-to-face interaction.

The findings differ to work by Hudson, ${ }^{19}$ who argued that there must be a 'strategy of planning for joint working' between collaborators. However, this study found that successful collaboration emerged organically through formal and informal social interactions mediated by co-location or professional organisations. GPs in this study reported that collaboration would only be successful with co-collaborators with similar 'health scientific backgrounds' which they conceptualised as 'credibility' and underpinned by trust. This speaks to McDonough and Doucette's ${ }^{6}$ 'norm development' concept and Howard et al $\mathrm{s}^{16}$ work which argued differences in 'role expectation' was a barrier to collaboration. Shared perspectives and paradigmatic norms about 'credibility', 'responsibility' and 'roles' can be renegotiated or changed through regular, social interaction. ${ }^{20}$ Other models describe better collaboration from a sense of 'knowing' or trust, and report perceived credibility 'building over time, 7172021 that concords with the findings from this study.

Models developed from empiric data have also shown that frequent exposure and interaction between professionals as 'directly influencing' collaboration, highlighting the value of "pharmacist and physician contact during training, ${ }^{13} \quad 14 \quad 17$ This supports our model as face-to-face interactions during training could lead to the construction of shared perspectives and trust.

Perceptions constructed through interaction might also allow previously held perspectives to change and trust to develop, overcoming barriers to collaboration such as the 'shopkeeper' stereotype. ${ }^{15}$ GPs in this study appeared to use 'demarcation strategies' in an 'attempt to reinforce medical dominance' by denouncing the qualifications, values and credibility of practitioners that held a 'different view of the world' or when improving adherence is linked to financial or commercial interests. ${ }^{715}$ From the pharmacists' perspective, there was an reluctance to contact GPs about non-adherence, adopting an apologetic tone and only contacting GPs as a reaction to an error, with a perception that dealing with adherence is not of interest to the GP. With increased social interaction, reported as sharing mobile phone numbers and contacting each other out of hours, attending functions together and camaraderie, these perspectives or 'world views' were able to change and trust develop.

Pharmacists and GPs in this study reported the best collaboration to improve adherence took place when
Figure 3 Barriers to successful collaboration to identify and improve non-adherence.

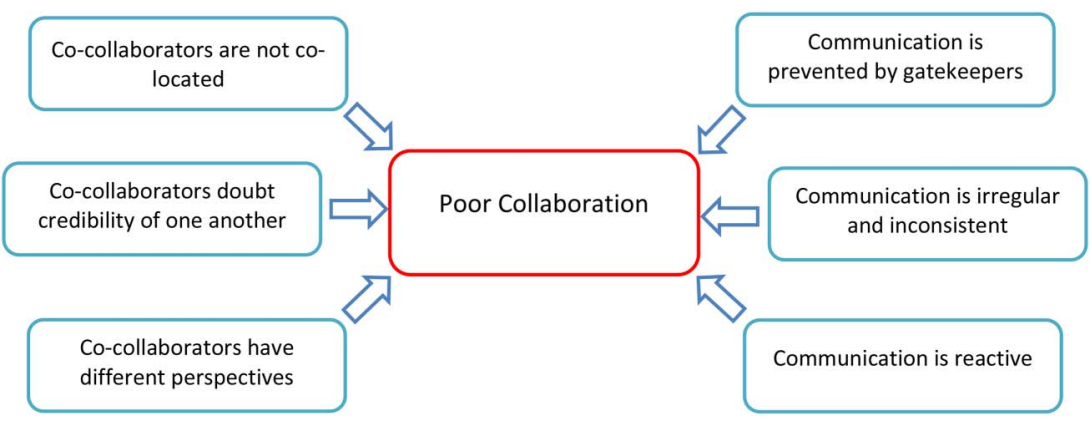


informal interactions occurred between the two professional groups regularly. As pharmacists and GPs engaged in informal, face-to-face and regular interaction, collaborations organically emerged as trust was developed as perceptions were changed and shared. This study theorises that shared perspectives and trust constructed through regular, face-to-face interaction, embedded by co-location, is essential for successful interprofessional collaboration to improve adherence.

In light of recent funding arrangements in the UK and proposals in Australia that will attempt to co-locate pharmacists in GP surgeries, ${ }^{22}{ }^{23}$ this work provides a theoretical basis for applications by clinicians, other healthcare professionals or researchers seeking funding. This work also offers practitioners insight into the functions of collaboration. The findings may be transferable to other settings and impact approaches to collaborations between other professional groups in primary or secondary care. Quantitative methods should be used to investigate the use of the conceptualised model as a theoretical framework underpinning interventions for increased and improved interprofessional collaboration to support adherence.

\section{Limitations}

In interpreting the findings, it should be noted that due to the qualitative nature of the study, the results may not be generalisable to a broader population of pharmacists and physicians, specifically those in a rural setting (as the participants in the study worked in a metropolitan area). Furthermore, a convenience sampling and recruitment process was utilised, which together with the reimbursement provided during the study, may imply that a biased sample may have participated in the focus groups. However, the recruitment ensured that there were approximately equal numbers of GPs and pharmacists of both genders who worked in a range of sociodemographic areas. Additionally, data collected were self-reported, and as such are limited by the accuracy of information provided by the respondents.

\section{CONCLUSION}

This work presents a novel conceptualisation of mediators of interprofessional collaboration to support patients' adherence to medications. Adherence-related interactions between community pharmacists and GPs do exist, but they are infrequent and limited. Pharmacists and GPs should attempt to develop regular, face-to-face interactions with each other to foster successful interprofessional collaboration. These findings may be transferable to different settings, such as collaborations between other healthcare professionals targeting different interventions.

Acknowledgements The authors would like to thank the pharmacists and GPs for taking part.
Contributors APR conducted secondary thematic analysis of data collected by SMM, PA, KH and IK. The data analysis was checked and discussed with SMM, PA and IK. APR prepared the first draft which was edited and approved by all authors.

Funding APR was supported by Durham University and AstraZeneca PIc to visit Australia.

Competing interests APR reports grants from AstraZeneca PIc and Durham University, during data analysis and manuscript writing, and grants from AstraZeneca PIc, outside the submitted work.

Ethics approval University of Sydney Human Research Ethics Committee (number 2013/176).

Provenance and peer review Not commissioned; externally peer reviewed.

Data sharing statement No additional data are available.

Open Access This is an Open Access article distributed in accordance with the Creative Commons Attribution Non Commercial (CC BY-NC 4.0) license, which permits others to distribute, remix, adapt, build upon this work noncommercially, and license their derivative works on different terms, provided the original work is properly cited and the use is non-commercial. See: http:// creativecommons.org/licenses/by-nc/4.0/

\section{REFERENCES}

1. Haynes RB, Ackloo E, Sahota N, et al. Interventions for enhancing medication adherence. Cochrane Database Syst Rev 2008;(2): CD000011.

2. Watts G. Doctors told to collaborate with community pharmacists to improve pain management. BMJ 2012;344:e350.

3. Bissell $\mathrm{P}$, Anderson C, Bacon L, et al. Community pharmacy supply of emergency contraception: impact of emergency contraception on women's and men's behaviour requires further exploration. BMJ 2001;323:751-1.

4. Mansoor SM, Krass I, Aslani P. Multiprofessional interventions to improve patient adherence to cardiovascular medications. J Cardiovasc Pharmacol Ther 2013;18:19-30.

5. Costa D, Van C, Abbott $P$, et al. Investigating Facilitators of GP engagement with Home Medicines Review (HMR): psychometric properties of the Home Medicines Review Inventory (HMRI). $J$ Interprof Care 2015;29:469-75.

6. McDonough R, Doucette W. Developing collaborative working relationships between pharmacists and physicians. J Am Pharm Assoc (2003) 2001;41:682-92.

7. Bradley F, Ashcroft DM, Noyce PR. Integration and differentiation: a conceptual model of general practitioner and community pharmacist collaboration. Res Soc Admin Pharm 2012;8:36-46

8. Leathard A. Models for interprofessional collaboration. East Sussex: Brunner-Routledge, 2003.

9. Leathard A. Going inter-professional: working together for health and welfare. London: Routledge, 1994.

10. Hudson B. Pessimism and optimism in inter-professional working: the Sedgefield Integrated Team. J Interprof Care 2007;21:3-15.

11. Van C, Costa D, Mitchell B, et al. Development and initial validation of the pharmacist frequency of interprofessional collaboration instrument (FICl-P) in primary care. Res Soc Admin Pharm 2012;8:397-407.

12. Van C, Costa D, Mitchell B, et al. Development and validation of a measure and a model of general practitioner attitudes toward collaboration with pharmacists. Res Soc Admin Pharm 2013;9:688-99.

13. Van C, Costa D, Mitchell B, et al. Development and validation of the GP frequency of interprofessional collaboration instrument (FICI-GP) in primary care. J Interprof Care 2012;26:297-304.

14. Van $C$, Costa $D$, Abbott $P$, et al. Community pharmacist attitudes towards collaboration with general practitioners: development and validation of a measure and a model. BMC Health Serv Res 2012;12:320.

15. Hughes C, McCann S. Perceived interprofessional barriers between community pharmacists and general practitioners: a qualitative assessment. Br J Gen Pract 2003;53:600-6.

16. Howard M, Trim K, Woodward C, et al. Collaboration between community pharmacists and family physicians: lessons learned from the Seniors Medication Assessment Research Trial. J Am Pharm Assoc (2003) 2003;43:566-72.

17. Bardet J, Thi-Ha V, Bedouch $P$, et al. Physicians and community pharmacist collaboration in primary care: a review of specific models. Res Soc Admin Pharm 2015;11:602-22. 
18. Crotty M. The foundations of social research: meaning and perspective in the research process. Sydney: SAGE Publications Ltd., 1998.

19. Hudson B. Joint working. Prospects of partnership. Health Serv J 1998;108:26-7.

20. Berger PL, Luckmann T. The social construction of reality: a treatise in the sociology of knowledge. London: Penguin Books, 1966.
21. Doucette WR, Nevins J, McDonough RP. Factors affecting collaborative care between pharmacists and physicians. Res Soc Admin Pharm 2005;1:565-78.

22. NHS England. New $£ 15 m$ scheme to give patients pharmacist support in GP surgeries. Redditch: NHS England, 2015.

23. Australian Medical Association. General Practice PharmacistsImproving Patient Care. 2015. 